\title{
Development of a landmark following type autonomous mobile robot using image processing
}

\author{
Naoyuki Ishijima ${ }^{\mathrm{a},},{ }^{,}$, Yu Nakagawa ${ }^{\mathrm{b}}$, Seiichi Serikawa ${ }^{\mathrm{a}}$ \\ ${ }^{a}$ Kyushu Institute of Technology, 1-1 Sensui-cho Tobata-ku Kitakyushu-shi Fukuoka-ken, 804-8550, Japan \\ *Corresponding Author: o108014n@mail.kyutech.jp
}

\begin{abstract}
In recent years, the use of online mail ordering including Amazon and the like has increased worldwide, and it has become an indispensable part of daily life. Amazon is ordered 30 million items a day, and it delivered. In order to manage these inventories and transport them accurately, the robot moves autonomously and carries the baggage in the factory. In the conventional method, there are a line trace type robot which moves on the line that was drawn on the floor of the factory, and a robot which chases a large amount of a sticker which were set at regular intervals with bar code. The advantage of these is that it can follow the route exactly. As a disadvantage, when changing the route, it is necessary to peel off the line and paste it again or rework the program. In addition, there are limitations when some stickers deteriorate and a robot is inability to move freely by some obstacles on the line. And there is a disadvantage that the use place is limited in common to these autonomous mobile robots.

Therefore, by developing an active landmark using red light and an autonomous mobile robot that images the image from the camera and chase the landmark, it solves the conventional problem by omitting labor and cost at the time of route change I thought that I could do. In this research, we aim to develop landmarks that actively send and receive signals by themselves as fundamental research, and turn on and off in red light.
\end{abstract}

\section{Introduction}

In recent years, the use of online mail ordering including Amazon and the like has increased worldwide, and it has become an indispensable part of daily life. People all over the world can easily order what they want using the internet and a system that carries goods to your home in a few days has been established. To support these convenient systems, it is necessary to manage and accurately inventory a huge amount of goods. Therefore, in the factory, the transport robot moves autonomously and carries the baggage.

Several methods can be cited as a conventional method for autonomous movement.

The first method is a line trace type robot that moves along the line drawn on the floor of the factory, the second method is a method in which a large amount of seal with a bar code is stuck on the floor at regular intervals and the robot moves along it, the third method is the robot scans surrounding circumstances with a laser and gathers data to create a room map.

The first method is easiest. The merit of the first method and the second method is that it can follow the route accurately. The disadvantage is that when you change the route you need to peel off the line and paste it again or rework the program. Also concern about deterioration of the seal. In addition, even if restrictions are imposed such as not placing objects on the line in the factory, the probability is not 0 , so it is impossible to move by a free route such as detouring when there is an object. The disadvantage of the third method is that it is troublesome because it is necessary to take data again with a laser when changing the route. In common with these robots, there is a disadvantage that the place of use is limited.

Also, irregular situations such as people's movements and existence of luggage may occur in human-only factories and workers who work together with humans and robots. At that time, it can not cope with the conventional method. So, 
it is difficult to realize by conventional methods in order to operate in any environment.

As robots can freely change obstacles and destinations like cars and move accurately to the destination even if they deviate from the set route, it is difficult with the conventional method, so a more flexible mobile system I have to think.

Towards the development of a new autonomous mobile system, looking up the common points of the existing methods, the following common points were found. That is that landmarks are passive signs that do not transmit signals or data themselves. Since it is a passive landmark, it is difficult to change the route by himself or to control something by sending a signal to the robot side. Therefore, if landmarks themselves become active markers to transmit signals and data themselves, we thought that improvement of existing autonomous mobile system could be achieved.

Therefore, by developing an active landmark using red light and an autonomous mobile robot that performs image processing on the image from the camera and follows the landmark, aimed to solve the problem of the conventional method by omitting labor and cost when changing the route. In this research, we aim to develop landmarks that actively send and receive signals by themselves as fundamental research, and turn on and off in red light.

\section{Principle}

\subsection{Arduino}

Arduino refers to a system composed of an infrastructure equipped with an AVR microcomputer and an input / output port, own Arduino language, Integrated development environment. Designed and manufactured by Arduino LLC and Arduino SRL. AVR microcomputer is a generic term for the 8-bit microcontroller product group manufactured by Atmel. Since Arduino is a microcomputer board, it is good at motor control, LED control, serial communication.

Arduino UNO R3 was used in this reserch. The Arduino UNO R3 is equipped with the ATmega 328 chip, which is characterized by having what is necessary to operate the microcontroller. In the following we will omit Arduino UNO R 3 and make it Arduino. The operating voltage is $5 \mathrm{~V}, 14$ digital I / O pins and 6 analog input pins. Wired serial communication is possible via the Arduino USB port, but the 0 th pin and 1st pin of the digital I / O pin can also perform serial communication and can be assigned to $\mathrm{RX}$ (transmission) TX (reception) respectively.

\subsection{Xbee}

Xbee is a radio module compliant with the ZigBee standard and is a product of Digi International. In this research, Xbee PRO S2B was used. Xbee PRO S2B is characterized by longer radio reach than normal Xbee. In the following, Xbee PRO S2B is omitted to be Xbee. In Japan, only models that use the same $2.4 \mathrm{GHz}$ radio band as $\mathrm{Wi}-\mathrm{Fi}$ and Bluetooth can be used. The ZigBee standard is one of the short range wireless communication standards. Physical layer and data link layer conform to IEEE 802.15.4. Although its communication speed is not so fast, its power consumption is extremely small, and it is suitable for constructing a large-scale network exceeding several hundred units.

\section{System configuration}

The conceptual diagram of the system of this research is shown in Fig. 3. The model proposed in this research consists of a total of three devices, two landmarks that can communicate with each other and one Xbee. The landmark 1 serves as a master unit that plays the central role of wireless communication and the other devices form a star type network that acts as a slave unit. Xbee is used as a substitute for the communication unit of the autonomous mobile robot to be developed in the future. Connect this Xbee to the PC and check the communication result on the serial monitor.

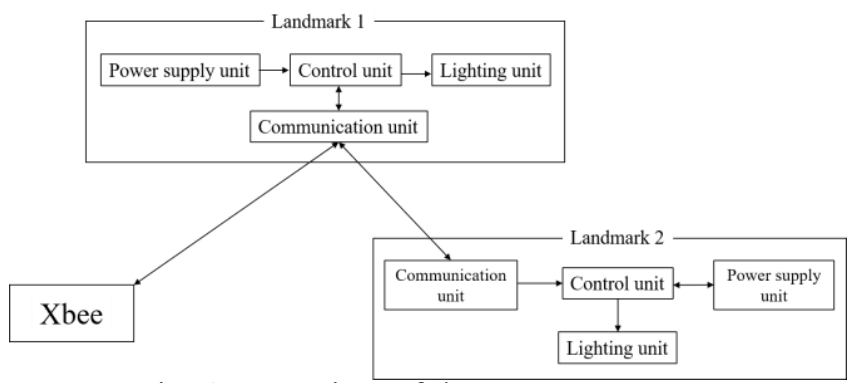

Fig. 1. Overview of the system

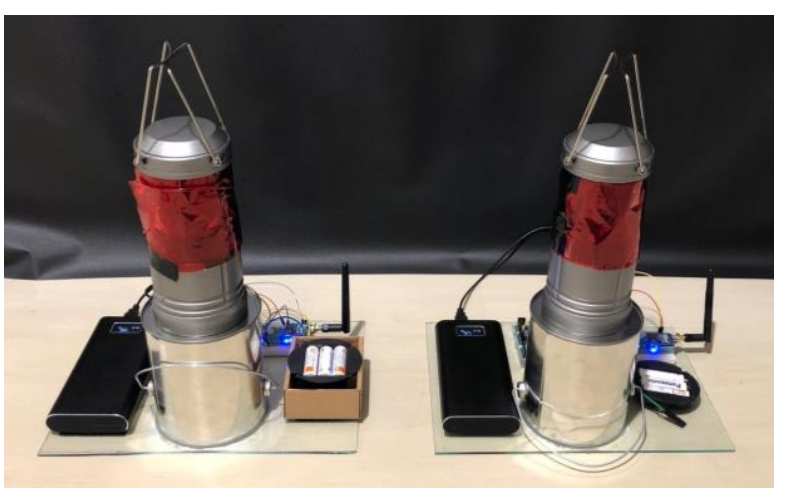

Fig. 2. A complete view of the Landmark 


\subsection{Landmark 1}

The structure of the landmark 1 is classified into four major types, "control unit", "communication unit", "lighting unit", and "power supply unit". The control unit is composed of Arduino UNO 3. In the communication section, wireless communication by Xbee is used. In the light section, a red cellophane is wrapped around a commercially available LED lantern. The power supply unit uses a mobile battery. Landmark 1 plays a central role in this autonomous mobile system.

Next, the operation of Arduino mounted on landmark 1 is explained. Control of red light and wireless communication by Xbee are done by Arduino. Landmark 1 receives an instruction from Xbee, turns itself on and off, and turns on and off landmark 2.

Next, the communication unit of the landmark 1 will be described. The communication section used Xbee. Xbee mounted on Landmark 1 is set as a coordinator (master unit) that communicates in AT mode, and the address of the destination is set to broadcast of 0xFFFF, so signals transmitted from the master unit are transmitted to all slave units that have set the same PAN. Communication power is supplied from Arduino.

Next, the lighting part of the landmark 1 will be described. LED lantern was used for the lighting part. This lantern is a mechanism that automatically turns on the LED when pulling out the lighting part with a handle, so by turning on the wire inside and letting the $5 \mathrm{~V}$ relay through, the LED lantern turns on and off with the signal from Arduino I made it possible to switch.

In the power supply section of the landmark 1, a mobile battery was used.

\subsection{Landmark 2}

The structure of the landmark 2 is classified into four major types, "control unit", "communication unit", "lighting unit", and "power supply unit". The control unit is composed of Arduino UNO 3. In the communication section, wireless communication by Xbee is used. In the light section, a red cellophane is wrapped around a commercially available LED lantern. The power supply unit uses a mobile battery. The landmark 2 plays a role of a mobile device operating with a signal from the landmark 1.

The control unit of the landmark 2 will be described. The main function of the control unit is to repeat turning on and off the LED according to the signal from the landmark 1. Arduino was used to realize this function.
Next, the communication unit of the landmark 2 will be described. The communication section used Xbee. Xbee installed on the landmark 2 is set as a router (child machine) to communicate in the AT mode, so that it can transmit a signal only to the landmark 1 which is the parent machine.

The lighting part and the power source part have the same configuration as the landmark 1 .

\section{Experiments and result}

Experiment using two landmarks and Xbee to send and receive only signals instead of robots.

\subsection{Preliminary experiment}

Before starting the experiment, we measured the communicable distance of Xbee as a preliminary experiment. If you set up the equipment apart from the communicable distance and do experiments, the experiment will fail.

First, we measured the communication distance in case of good visibility. The measurement results of the communicable distance are shown in Table 1. Connect the Xbee of the parent machine to the PC and check whether the communication is normally done by the XCTU serial monitor. Let Xbee of the slave unit separate from the master unit and measure the distance that communication can not be performed. When communication is possible, the characters sent by the serial monitor are returned as they are. When communication is impossible, only sent letters are displayed. Blue indicates transmission data, and red indicates reception data.

Table 1 result of communicable distance

\begin{tabular}{|c|c|}
\hline $\begin{array}{c}\text { Communication } \\
\text { distance }\end{array}$ & Display of serial monitor \\
\hline $1 \mathrm{~m}$ & aa \\
\hline $3 \mathrm{~m}$ & aa \\
\hline $5 \mathrm{~m}$ & aa \\
\hline $10 \mathrm{~m}$ & aa \\
\hline $15 \mathrm{~m}$ & aa \\
\hline $20 \mathrm{~m}$ & aa \\
\hline $25 \mathrm{~m}$ & aa \\
\hline $30 \mathrm{~m}$ & aa \\
\hline $35 \mathrm{~m}$ & aa \\
\hline $40 \mathrm{~m}$ & aa \\
\hline $45 \mathrm{~m}$ & aa \\
\hline $50 \mathrm{~m}$ & aa \\
\hline $55 \mathrm{~m}$ & aa \\
\hline $60 \mathrm{~m}$ & aa \\
\hline
\end{tabular}


Because the limit length of the building where the experiment was conducted was $60 \mathrm{~m}$, we could only do the experiment up to $60 \mathrm{~m}$. Since the experiment is conducted in the case of good prospects in this research, if communication is possible at $60 \mathrm{~m}$, it will not cause any trouble.

\subsection{Experimental study on active landmark}

Check whether two landmarks operate as active landmarks. Experiments are conducted using two landmarks and one Xbee machine. Xbee is used as a substitute for the robot following the landmark. The transmission and reception of the signals of the robot and the landmark will be described.

When the robot follows the landmark, a tracking completion signal is sent from the robot to the landmark1. When the landmark 1 is lit when receiving the arrival signal, the landmark 1 is turned off and the lighting signal is transmitted to the landmark 2. Then, the following restart signal is transmitted to the robot. When the landmark 2 is lit when receiving the arrival signal, an extinguish signal is transmitted to the landmark 2 and the landmark 1 is lit. Then, the following restart signal is transmitted to the robot. Also, after tracking is completed, the follow-up completion signal is transmitted to the robot, and the process is ended.

Table 2 shows combinations of signal transmission and reception.

Table 2 combinations of signal

\begin{tabular}{|c|c|c|c|c|}
\hline & Xbee & & Landmark1 & Landmark2 \\
\hline \multirow{3}{*}{ (1) } & Send 'S' & & Lights off & \\
\hline & & & Send ' 0 ' & $\rightarrow$ Lighting up \\
\hline & $\begin{array}{l}\text { Follow-up } \\
\text { resumption }\end{array}$ & & Send ' 8 ' & \\
\hline \multirow{3}{*}{ (2) } & Send 'S' & & Lighting up & \\
\hline & & & Send ' 1 ' & $\rightarrow$ Lights off \\
\hline & $\begin{array}{l}\text { Follow-up } \\
\text { resumption }\end{array}$ & & Send ' 8 ' & \\
\hline \multirow{2}{*}{ (3) } & Send 'S' & - & Lights off & Lights off \\
\hline & $\begin{array}{l}\text { Follow-up } \\
\text { completion }\end{array}$ & & Send ' 9 ' & \\
\hline
\end{tabular}

Explain the type of signal. Xbee is an alternative to autonomous mobile robot, connecting to $\mathrm{PC}$ and sending and receiving signals. (1) is the pattern where landmark 1 is lit first, (2) is the pattern where landmark 2 is lit first, (3) is a pattern that follows all the landmarks. ' $\mathrm{S}$ ' is a follow-up completion signal sent from the robot, and it is sent when following the landmark. After $\mathrm{S}$ is sent, Landmark 1 conducts exchange of all signals . ' 0 ' is a signal for turning on the landmark 2. ' 1 ' is a signal for turning off the landmark
2. 8 is a signal for causing the robot to resume tracking. 9 is a signal to end the tracking of the robot. By these signals, it is confirmed whether the landmark actively operates.

As a result of the experiment, it was confirmed that landmarks 1 and 2 are turned on and off when $\mathrm{S}$ is sent in both patterns (1) and (2). And I could also confirm that I sent a follow-up restart signal to Xbee. In the case of pattern (3), it was confirmed that both landmarks 1 and 2 were turned off, and I could also confirm that I sent a tracking end signal to Xbee. In the future we will replace this Xbee with a robot and complete the system.

\section{Examination of results}

From the experiment in 4.1, we found that wireless communication is carried out up to $60 \mathrm{~m}$. From the experimental results, it was shown that in this research it is possible to reliably communicate without considering the communicable distance.

Moreover, in the experiment of 4.2, it was confirmed that the landmark operates as an active landmark. In this experiment, the number of devices was small, there were only two patterns of lighting patterns from landmark 1 to landmark 2, or landmark 2 to landmark 1 . Therefore, it seems easy to decide the route, but as the number of equipment increases, it becomes difficult to decide the route. However, as in this experiment, it can be considered that it is possible to easily determine the route by using the landmark 1 as the parent machine. In addition, since landmarks can be easily carried around, changing the route is much simpler than conventional methods.

From the above, it was possible to realize ease of route change which was impossible by conventional method by developing active landmark.

\section{Conclusion}

In this research, we were able to achieve the goal of developing active landmarks among the entire autonomous mobile systems that were the original objectives. As a result of device development and operation experiment, we showed that the landmark has turned on and turned off by transmitting and receiving signals. And it became possible to flexibly change the route. Moreover, it showed that landmark 1 became the parent machine, and it was possible to gather information from all the devices and issue instructions. The system developed this time is more effective when the number of devices increases. It showed that we were able to 
develop a flexible autonomous mobile system that has never been done since we followed the device in any way.

In the future, to complete the system, it is necessary to develop an autonomous mobile robot that uses image processing to follow this landmark and actually follow it.

\section{References}

(1) Yasuhiro Kanzaki : "Measure, measure, weigh in Arduino", pp. 1-94, 2012 